\title{
LA INOCULACIÓN CON HONGOS MICORRÍCICO ARBUSCULARES PROMUEVE EL CRECIMIENTO DE PLANTINES DE KAGENECKIA LANCEOLATA (ROSACEAE)
}

\author{
SILVIA E. NAVARRO RAMOS ${ }^{1}$, DANIEL RENISON¹ y ALEJANDRA G. BECERRA²
}

\begin{abstract}
Summary: Inoculation with arbuscular mycorrhizal fungi promotes the growth of Kageneckia lanceolata (Rosaceae) seedlings. Arbuscular mycorrhizal fungi (AMF) increase the survival and biomass production of host plants and improve nutrient absorption. AMF are very important in the restoration of degraded soils and in the growth of vegetation in nursery and field conditions. In order to restore populations of Kageneckia lanceolata, species considered vulnerable by the World Conservation Monitoring Center, it was proposed to evaluate the effect of inoculation with AMF on the growth, radical colonization and nutritional content in this species. We performed a greenhouse experiment to test the hypothesis that inoculation with AMF promotes the growth and nutrition of $K$. lanceolata. The inoculum used was natural soil coming from the rhizosphere of a forest with $K$. lanceolata. After 6 months, plants inoculated with AMF showed twice more aerial biomass and three more phosphorus than non-inoculated seedlings. This is the first study that shows the positive effect of AMF on growth and mineral nutrition of $K$. lanceolata.
\end{abstract}

Key words: Durazno de campo, greenhouse, natural mycorrhizal soil inoculum, phosphorus, restoration, vulnerable species.

\begin{abstract}
Resumen: Los Hongos Formadores de Micorrizas Arbusculares (HFMA) incrementan la supervivencia y producción de biomasa de las plantas hospedadoras y mejoran la absorción de nutrientes. Los HFMA son muy importantes en la restauración de suelos degradados y en facilitar el crecimiento de la vegetación bajo condiciones de vivero y campo. Con el fin de restaurar las poblaciones de Kageneckia lanceolata, especie considerada vulnerable por World Conservation Monitoring Center, en este trabajo se propuso evaluar el efecto de la inoculación con HFMA en el crecimiento, la colonización radical y el contenido nutricional de esta especie. Para poner a prueba la hipótesis de que la inoculación con los HFMA promueve el crecimiento y la nutrición de $K$. lanceolata, se realizó un experimento de invernadero. El inóculo utilizado fue suelo natural proveniente de la rizosfera de un bosque con $K$. lanceolata. Después de 6 meses, los plantines inoculados con HFMA presentaron dos veces más de biomasa aérea y tres veces más de fósforo que los no inoculados. Este es el primer estudio que muestra el efecto positivo de los HFMA sobre el crecimiento y la nutrición mineral de $K$. lanceolata.
\end{abstract}

Palabras clave: Durazno de campo, especie vulnerable, fósforo, inóculo natural de micorrizas del suelo, invernadero, restauración.

\section{INTRODUCCIÓN}

Los Hongos Formadores de Micorrizas Arbusculares (HFMA) tienen gran importancia en la restauración de suelos degradados facilitando el crecimiento de la vegetación bajo condiciones de

${ }^{1}$ Centro de Ecología y Recursos Naturales RenovablesDr. Ricardo Luti (CERNAR-IIByT)-CONICET, UNC.

${ }^{2}$ Laboratorio de Micología, Instituto Multidisciplinario de Biología Vegetal (IMBIV)-CONICET, UNC. Contacto: silnara1@hotmail.com vivero y de campo (Turnau \& Haselwandter, 2002; Smith \& Read, 2008). El efecto positivo de los HFMA se puede observar en las plantas hospedadoras al incrementarse su reproducción, supervivencia y producción de biomasa (Jayachandran \& Fisher, 2008; Smith \& Read, 2008). Los HFMA mejoran la absorción de nutrientes (principalmente de fósforo) (Smith et al., 2011), la tolerancia a condiciones de estrés y la resistencia a los patógenos del suelo, entre otras funciones (Wehner et al., 2010; Millar \& Bennett, 2016). En especies leñosas nativas del centro de Argentina se ha probado la inoculación 
micorrícica en las especies Acacia caven, Polylepis australis y Prosopis chilensis, resultando positiva en las tres especies (Herrera et al., 1993; Pérez \& Urcelay, 2009; Soteras et al., 2013).

Sin embargo, los HFMA pueden tener un efecto neutro o negativo sobre la planta hospedadora, dependiendo de las condiciones ambientales y de las especies asociadas (Jones \& Smith, 2004; Smith \& Read, 2008). Tal es el caso de Fragara moschata y Potentilla reptans, ambas especies de la familia Rosaceae, que mostraron respuestas negativas en el crecimierto a la inoculación con HFMA (Sudová \& Vosátka, 2008). Debido a que el efecto de los HFMA no siempre es positivo, antes de llevar a cabo inoculaciones a gran escala con plantines destinados a ser usados en plantaciones, es conveniente realizar ensayos para determinar si estas inoculaciones son beneficiosas.

Kageneckia lanceolata Ruiz et Pavón. (Rosaceae, "durazno de campo") (de ahora en adelante Kageneckia) es una especie neotropical considerada vulnerable por World Conservation Monitoring Center (1998), siendo sus principales amenazas el pastoreo doméstico y la deforestación. Kageneckia es un árbol pequeño, de copa alargada, rala y estrecha, sus hojas son caducas, sus flores son imperfectas y se agrupan en cimas pequeñas y su fruto es leñoso en forma de estrella. Se distribuye en Perú, Bolivia y Argentina, encontrándose en el Chaco serrano y en su transición con las Yungas (Demaio et al., 2002). Hasta el momento no se han estudiado las interacciones Kageneckia-HFMA. Con el fin de ayudar a restaurar sus poblaciones y los ecosistemas donde se encuentra esta especie, en este trabajo se propuso evaluar, bajo condiciones de invernadero, el efecto de la inoculación con HFMA en el crecimiento, la colonización radical y el contenido nutricional en plantines de Kageneckia. Se pone a prueba la hipótesis de que la inoculación con los HFMA promueve el crecimiento y la nutrición de los plantines del durazno de campo.

\section{Material y Métodos}

\section{Área de estudio}

El distrito Chaqueño Serrano en la provincia de Córdoba, Argentina (lat. $-31,4^{\circ}$; long. $-64,6^{\circ}$ ), tiene una precipitación media anual de $859 \mathrm{~mm}$ y la temperatura media anual es de $15^{\circ} \mathrm{C}$. Entre las especies vegetales más representativas del lugar se pueden citar a Acacia caven, Celtis ehrenbergiana, Kageneckia lanceolata y Lithraea molleoides (Giorgis et al., 2005), siendo Kageneckia exclusiva del estrato arbóreo inferior del Chaco Serrano (Demaio et al., 2002).

\section{Material biológico utilizado}

Las semillas de Kageneckia utilizadas para la producción de plantines fueron recolectadas durante los meses de diciembre de 2013 y enero de 2014, de 9 árboles distribuidos en tres poblaciones de Córdoba (Argentina): Cuesta Blanca (lat.-31,481 ${ }^{\circ}$

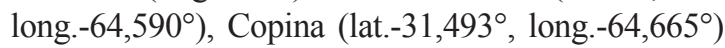
y Cerro Blanco (lat.-31,338 ${ }^{\circ}$, long.-64,664 ${ }^{\circ}$ ).

Las muestras de suelo utilizado para inocular las plántulas, se recolectaron de 12 bosques situados en los alrededores de la localidad de Cuesta Blanca (lat.31,459 ; long.-64,588 ). La recolección se realizó durante el invierno (agosto de 2014) de la rizosfera de Kageneckia (0-20 cm profundidad). Las muestras se colocaron en bolsas de polietileno, se mezclaron y almacenaron a $4^{\circ} \mathrm{C}$ hasta su procesamiento en el laboratorio. Las 12 muestras de suelo se homogenizaron para realizar los siguientes análisis edáficos: textura, nitrógeno total (\%) con el método de Kjeldahl (Bremner \& Mulvaney, 1982), materia orgánica (\%) (Walkley \& Black 1934), pH (25\% en solución con agua), fósforo extraíble (ppm) por medio del método de Bray and Kurtz I (Jackson, 1964), conductividad eléctrica, y la capacidad de intercambio catiónico (CIC).

A fin de conocer la diversidad de HFMA presentes en el inóculo, el suelo se tamizó con una malla de apertura de $1 \mathrm{~cm}$, para remover hojarasca, piedras y palos. Se tomaron $100 \mathrm{~g}$ de suelo rizosférico fresco que fue tamizado empleando mallas de distinto tamaño de apertura $(125$ y $38 \mu \mathrm{m})$, siguiendo la metodología propuesta por Gerdemann \& Nicolson (1963). El contenido retenido en los tamices fue centrifugado en sacarosa al 80\% (Walker et al., 1982). Las esporas obtenidas se colocaron en cápsulas de Petri de $9 \mathrm{~cm}$ de diámetro con base cuadriculada de $1 \mathrm{~cm}$ de lado. Las esporas y esporocarpos (aparentemente saludables y sin ningún tipo de ataque) fueron cuantificados e identificados a nivel de especie cuando fuera posible, bajo microscopio estereoscópico y microscopio óptico (Nikon, E200). Para cada tipo morfológico, se realizaron preparados permanentes usando polivinil-alcohol (PVA) y PVA 


\section{S. E. Navarro Ramos et al. - Crecimiento de Kageneckia lanceolata}

+ Melzer (Omar et al., 1979) como medios de montaje. Las morfoespecies fueron identificadas siguiendo los manuales de identificación de INVAM, Blaszkowski (2003), Schenk \& Perez (1990) y Redecker et al. (2013). Las esporas y esporocarpos de las morfoespecies fueron examinados y fotografiados con una cámara conectada a un microscopio Axiophot Zeiss (100 ISO).

\section{Inoculación de plantines de Kageneckia}

Las semillas de Kageneckia recolectadas se desinfectaron con hipoclorito de sodio al 10\% durante 10 min y se enjuagaron con agua esterilizada. Posteriormente, se germinaron en bandejas con arena esterilizada ( $1 \mathrm{~h}$ en autoclave a $120^{\circ} \mathrm{C}$ y $2 \mathrm{~atm}$ de presión). A los 3 meses, las plántulas fueron trasplantadas a macetas de $450 \mathrm{~g}$ con suelo natural tindalizado $\left(1 \mathrm{~h}\right.$ en autoclave a $100^{\circ} \mathrm{C}$, por 3 días consecutivos con intervalos de $24 \mathrm{~h}$ de reposo) y arena esterilizada en una proporción $3: 1$. El suelo natural tindalizado fue el mismo suelo utilizado para la obtención del inóculo.

Al momento del trasplante, se inocularon las plántulas con $100 \mathrm{~g}$ de suelo rizosférico fresco, localizado a $3 \mathrm{~cm}$ de la superficie. El tratamiento control consistió en $100 \mathrm{~g}$ de suelo rizosférico esterilizado, al que se le adicionaron $50 \mathrm{ml}$ de una solución de suelo nativo (100 g) previamente filtrado por un tamiz de $38 \mu \mathrm{m}$ a fin de excluir el inóculo micorrícico (esporas, micelio extraradical y fragmentos de raíces colonizados) y recuperar la microbiota nativa (Koide \& Li, 1989; Pérez \& Urcelay, 2009).

Los tratamientos fueron asignados aleatoriamente a las unidades experimentales, siguiendo un diseño completamente aleatorizado con 9 réplicas por cada uno de los tratamientos (inoculado y no inoculado).

Las condiciones de crecimiento en invernadero fueron de $25^{\circ} \mathrm{C}, 12 \mathrm{~h}$ de fotoperiodo y riego tres veces al día mediante riego automático por aspersión.

\section{Respuesta de Kageneckia frente a la inoculación}

$\mathrm{Al}$ final del experimento (6 meses) se cosecharon los 9 plantines de cada tratamiento y se midieron los parámetros de crecimiento vegetal: biomasa aérea y radical y el contenido de fósforo y de nitrógeno total en hojas. El contenido de fósforo total (\%) de las hojas se obtuvo por el método de digestión ácida (Jones et al., 1991) y $\mathrm{N}$ total (\%) de las hojas se obtuvo por el método Kjeldahl (volumetría ácido-base).
La colonización micorrícica arbuscular (CMA) fue evaluada siguiendo las técnicas descritas por Phillips \& Hayman (1970) y Grace \& Stribley (1991). Se utilizaron $0,5 \mathrm{~g}$ de raíces frescas las que se aclararon con $\mathrm{KOH}$ al $10 \%\left(15 \mathrm{~min}, 90^{\circ} \mathrm{C}\right)$, posteriormente se acidificaron con HCL al 1\% (1 min, temperatura ambiente) y tiñeron con Azul de Anilina al 0,05\% (5 min, $90^{\circ} \mathrm{C}$ ). Se cuantificó el porcentaje de CMA y de estructuras intra-radicales (arbúsculos, vesículas e hifas), siguiendo la técnica propuesta por McGonigle et al. (1990) utilizando un microscopio óptico (Nikon, E200) a 400x de aumento.

\section{Análisis de datos}

Las variables respuesta: biomasa aérea y radicular y el contenido de nutrientes en las hojas (fósforo y nitrógeno total) se compararon entre los dos tratamientos (inoculado y no inoculado) mediante métodos no paramétricos. En todos los casos se aplicó la prueba de Kruskal-Wallis. La correlación entre la biomasa aérea y el fósoforo se realizó con el coeficiente de correlación lineal de Pearson, con un nivel de significancia de 0,05 . Todos los análisis se realizaron utilizando el programa estadístico Infostat (Di Rienzo et al., 2015).

\section{Resultados}

Inóculo: características del suelo y comunidad de HFMA

El suelo de la rizofera de Kageneckia utilizado como inóculo se caracterizó por presentar una textura franco arenosa $(58,5 \%$ arena, $33,2 \%$ limo y $8,3 \%$ arcilla), un $\mathrm{pH}$ moderadamente ácido y una muy alta capacidad de intercambio catiónico. Además, una alta concentración de fósforo, contenido de nitrógeno total, porcentaje de materia orgánica y relación carbono:nitrógeno (Tabla 1).

La abundancia de esporas en el inóculo micorrícico fue de 120 esporas/100 g de suelo seco. Se identificaron 7 morfoespecies pertenecientes a 6 géneros (Acaulospora, Claroideoglomus, Funneliformis, Gigaspora, Glomus y Scutellospora): Acaulospora mellea Spain \& Schenck, Claroideoglomus claroideum Schenck \& Smith, Funneliformis mosseae (Nicolson \& Gerdemann) Walker \& Schuessler, Gigaspora margarita Becker \& Hall, Glomus brohultii Herrera, Ferrer \& Sieverding, Glomus fuegianum (Spegazzini) Trappe 
Tabla 1. Propiedades edáficas del suelo de la rizosfera de 12 árboles de Kageneckia lanceolata utilizado como inóculo.

\begin{tabular}{|cc|}
\hline $\begin{array}{c}\text { Tipo de medición y } \\
\text { unidades utilizadas }\end{array}$ & Valor \\
Clase textural & Franco-arenoso \\
\hline $\mathrm{pH}$ & 5,5 \\
\hline Materia Orgánica (\%) & 15,44 \\
\hline Carbono orgánico (\%) & 8,96 \\
\hline Nitrógeno total (\%) & 0,63 \\
\hline Relación Carbono:Nitrógeno & 14,2 \\
Fósforo (ppm) & 24,5 \\
\hline Conductividad Eléctrica (dS/m) & 3,7 \\
\hline Humedad equivalente (\%) & 32,7 \\
\hline $\begin{array}{c}\text { Capacidad de intercambio } \\
\text { catiónico (meq/100g) }\end{array}$ & 35,5 \\
\hline
\end{tabular}

\& Gerdemann, y Scutellospora biornata Spain, Sieverding \& Toro.

Crecimiento de los plantines y colonización micorrícica

Después de 6 meses de crecimiento en invernadero, los plantines inoculados presentaron dos veces más biomasa aérea y tres veces más fósforo que los no inoculados (Tabla 2, Fig. 1). Se observó una correlación lineal de Pearson con una tendencia positiva entre el fósforo y la biomasa aérea $(\mathrm{r}=0,334$; $\mathrm{P}=0,071)$. Los plantines inoculados presentaron un $12 \%$ más nitrógeno total que los no inoculados, mientras que la biomasa radical no difirió entre los plantines con y sin inóculo (Tabla 2, Fig. 1).

Los plantines control (no inoculados) no presentaron CMA, evidenciando ausencia de contaminación en el transcurso del experimento. La CMA en los plantines inoculados varió entre 80 y 95\%. Las estructuras micorrícicas presentes en los plantines inoculados fueron puntos de entrada, hifas, vesículas y arbúsculos (Fig. 2). La colonización de hifas varió de 47 a $78 \%$, la de vesículas de 11 a $43 \%$ y la de arbúsculos de $0-5 \%$.
Tabla 2. Biomasa aérea, biomasa radical y contenido de nutrientes en plantines inoculados y no inoculados de Kageneckia lanceolata. Se muestran las medias \pm error estándar de 9 muestras. Letras diferentes indican diferencias significativas $(P<0,05)$ según la prueba de Kruskal-Wallis.

\begin{tabular}{|ccccc|}
\hline & $\begin{array}{c}\text { Plantines } \\
\text { inoculados }\end{array}$ & $\begin{array}{c}\text { Plantines } \\
\text { no } \\
\text { inoculados }\end{array}$ & valor P & Prueba \\
\hline $\begin{array}{c}\text { Biomasa } \\
\text { aérea (g) }\end{array}$ & $5,54 \pm 0,70 \mathrm{a}$ & $2,34 \pm 0,62 \mathrm{~b}$ & 0,008 & $\mathrm{H}=6,79$ \\
$\begin{array}{c}\text { Biomasa } \\
\text { radical (g) }\end{array}$ & $1,11 \pm 0,12 \mathrm{a}$ & $0,73 \pm 0,19 \mathrm{a}$ & 0,114 & $\mathrm{H}=2,67$ \\
$\begin{array}{c}\text { Fósforo } \\
(\%)\end{array}$ & $0,17 \pm 0,01 \mathrm{a}$ & $0,05 \pm 0,01 \mathrm{~b}$ & $<0,0001$ & $\mathrm{H}=12,79$ \\
$\begin{array}{c}\text { Nitrógeno } \\
\text { total (\%) }\end{array}$ & $1,84 \pm 0,09 \mathrm{a}$ & $1,63 \pm 0,05 \mathrm{a}$ & 0,053 & $\mathrm{H}=3,77$ \\
\hline
\end{tabular}

\section{Discusión y Conclusiones}

La inoculación de especies leñosas con HFMA ha sido puesta a prueba en algunos trabajos a fin de optimizar la restauración de zonas degradadas (Allen et al., 2003; Caravaca et al., 2005).

Los HFMA son importantes en la absorción de nutrientes y en el incremento del crecimiento de muchas especies de plantas (Jeffries et al., 2003; Smith \& Read 2008). En este trabajo la inoculación con HFMA de plantines de Kageneckia bajo condiciones de invernadero resultó ser efectiva. Esto apoya nuestra hipótesis y coincide con otros trabajos llevados a cabo en invernadero (Ouahmane et al., 2007; Echairi et al., 2008; El Mrabet et al., 2014). Se observó una alta cantidad de fósforo en los tejidos de los plantines inoculados con HFMA lo que puede explicar el aumento de la biomasa aérea, además una correlación con tendencia positiva entre ambas variables. Esto coincide con los resultados obtenidos por El Mrabet et al. (2014) para plantines de Argania spinosa. A diferencia de otros ensayos de invernadero donde el inóculo micorrícico promovió un aumento en la biomasa radical de $P$. australis (Soteras et al., 2013), en este trabajo no se observó este mismo efecto en las raíces de Kageneckia. Esto coincide con la teoría de asignación de biomasa que sugiere que la colonización micorrícica disminuye la relación raíz/tallo, ya que las plantas micorrizadas 


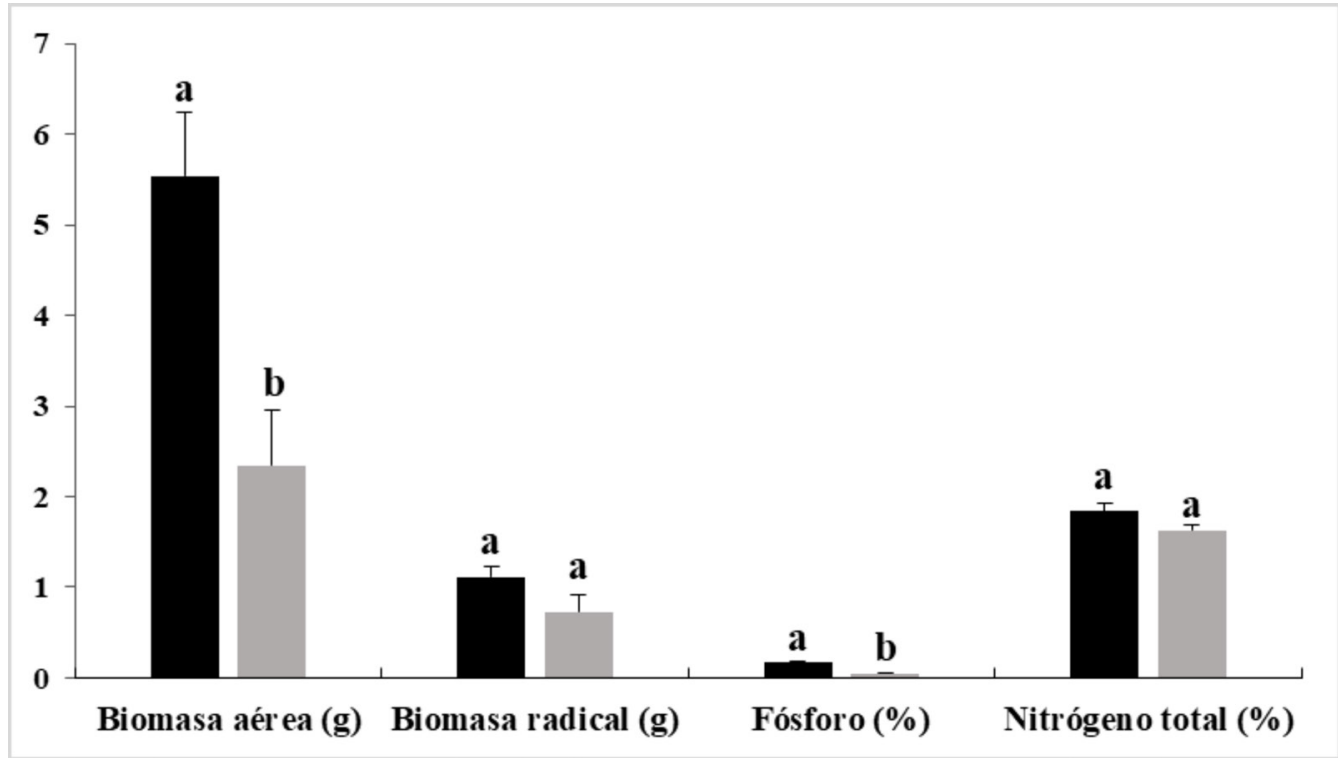

Fig. 1. Biomasa aérea, biomasa radical y porcentajes de nutrientes (fósforo y nitrógeno total) en plantines inoculados y no inoculados ( $\square$ : inoculados; $\square$ : no inoculados) de Kageneckia lanceolata después de 6 meses de crecimiento. Se indica la media \pm error estándar de 9 muestras. Letras diferentes indican diferencias significativas $(P<0,05)$ entre los dos tratamientos mediante la prueba de Kruskal-Wallis.

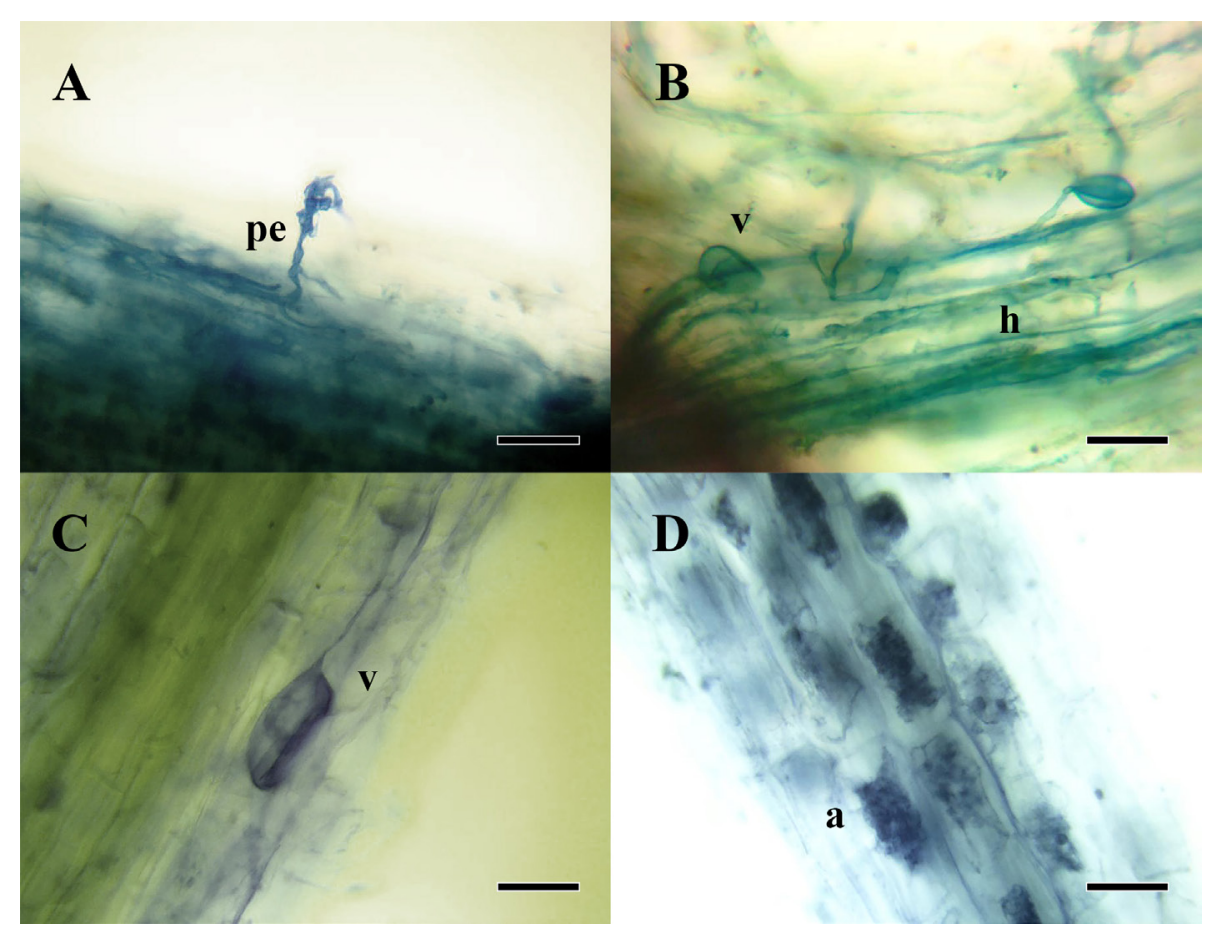

Fig. 2. Estructuras micorrícicas en raíces colonizadas de Kageneckia lanceolata. A: Punto de entrada (pe). B: Hifas (h) y vesículas (v). C: Vesículas (v). D: Arbúsculos (a). Escala A-D: 10 m. 
asignan menos fotosintatos al crecimiento radical al adquirir los nutrientes principalmente a través del micelio extraradical de los HFMA asociados con sus raíces (Johnson 2010; Veresoglou et al., 2011).

Las esporas del inóculo micorrícico utilizado, coinciden con los géneros encontrados en la rizosfera de otras especies de la familia Rosaceae (Błaszkowski, 1994; Soteras et al., 2013). Los inóculos de primavera en general presentan un mayor número de esporas en el suelo (mayor diversidad y riqueza), lo que se traduce en un mayor crecimiento del árbol (Lugo \& Cabello 2002; Soteras et al., 2014). En este estudio usamos inóculo de invierno que también resultó exitoso en cuanto al crecimiento y colonización micorrícica de Kageneckia.

Este trabajo muestra por primera vez, bajo condiciones de invernadero, que Kageneckia se micorriza y que requiere de los HFMA para su crecimiento y nutrición mineral. A fin de optimizar prácticas en restauración, resultaría importante evaluar el crecimiento de plántulas de Kageneckia con y sin inóculo bajo condiciones de campo.

\section{Agradecimientos}

Este trabajo fue financiado por la Agencia de Promoción Científica y Tecnológica- PICT 2013049. Agradecemos al Biól. Nicolás Marro por su ayuda en el cuidado de los plantines.

\section{Bibliografía}

ALLEN, E. B., M. F. ALLEN, L. EGERTONWARBURTON, L. CORKIDI \& A. GÓMEZPOMPA. 2003. Impacts of early- and late-seral mycorrhizae during restoration in seasonal tropical forest, Mexico. Ecol. Appl.13: 1701-1717.

BLASZKOWSKI, J. 2003. Arbuscular mycorrhizal fungi (Glomeromycota), Endogone and Complexipes species deposited in the Departament of Plant Pathology, Univerisity of Agriculture in Szczecin, Poland. Disponible en: http://www.zor.zut.edu.pl/ Glomeromycota/Taxonomy.html [Acceso: 25 Julio 2017].

BREMNER, J. M. \& C. S. MULVANEY. 1982. Nitrogentotal. In: PAGE, A. L. (ed.), Methods of soil analysis Part II, pp. 562-595. American Society of Agronomy: Soil Science Society of America, Madison, Wisconsin.
CARAVACA, F., M. M. ALGUACIL, J.M. BAREA \& A. ROLDÁN. 2005. Survival of inocula and native AM fungi species associated with shrubs in a degraded Mediterranean ecosystem. Soil Biol. Bioch. 37: 227-233.

DEMAIO, P., U.O. KARLIN \& M. MEDINA. 2002. Árboles nativos del centro de Argentina. L.O.L.A. Buenos Aires.

DI RIENZO J. A., F. CASANOVES, M. G. BALZARINI, L. GONZALEZ, M. TABLADA, C. W. ROBLEDO. InfoStat versión 2015. Grupo InfoStat, FCA, Universidad Nacional de Córdoba, Argentina. Disponible en: http://www.infostat. com.ar.

ECHAIRI, A., R. NOUAIM, \& R. CHAUSSOD. 2008. Intérêt de la mycorhization contrôlée pour la production de plants d'arganier (Argania spinosa) en conditions de pépinière. Sécheresse 19: $277-$ 281 .

EL MRABET S., L. OUAHMANE, A. EL MOUSADIK, F. MSANDA \& Y. ABBAS. 2014. The effectiveness of arbuscular mycorrhizal inoculation and biocompost addition for enhancing reforestation with Argania spinosa in Morocco. Open Journal of Forestry 4: 14-23.

GERDEMANN, J. W. \& T. H. NICOLSON. 1963. Spores of a mycorrhizal Endogone species extracted from the soil by wet sieving and decanting. Trans. Br. Mycol. Soc. 46: 235-244.

GIORGIS, M. A., A. M. CINGOLANI, D. E. GURVICH, N. REYNERO \& S. RUFINI. 2005. Diferencias en la estructura de la vegetación del sotobosque entre una plantación de Pinus taeda L. (Pinaceae) y un matorral serrano (Cuesta Blanca, Córdoba). Kurtziana 31.

GRACE, C. \& D. P. STRIBLEY. 1991. A safer procedure for routine staining of vesicular-arbuscular mycorrhizal fungi. Mycol. Res. 95: 1160-1162.

HERRERA, M. A., C. P. SALAMANCA \& J. M. BAREA. 1993. Inoculation of woody legumes with selected arbuscular mycorrhizal fungi and rhizobia to recover desertified Mediterranean ecosystems. Appl. Environ. Microb. 59: 129-133.

INVAM (International Collection of VesicularArbuscular Mycorrhizal Fungi). Disponible en http://invam.caf.wvu.edu/fungi/taxonomy/ speciesID.htm [Acceso: 25 Julio 2017].

JACKSON, M. L. 1964. Análisis químico de suelos. 2nd Edn. Omega, Barcelona.

JAYACHANDRAN, K. \& J. FISHER. 2008. Arbuscular mycorrhizae and their role in plant restoration in native ecosystems. In: SIDDIQUI, Z. A. \& K. FUTAI (eds.), Mycorrhizae: Sustainable Agriculture and Forestry, pp. 195-209. New Delhi. Springer. 


\section{S. E. Navarro Ramos et al. - Crecimiento de Kageneckia lanceolata}

JEFFRIES, P., S. GIANINAZZI, S. PEROTTO, K. TURNAU \& J. M. BAREA. 2003. The contribution of arbuscular mycorrhizal fungi in sustainable maintenance of plant health and soil fertility. Biol. Fert. Soils. 37: 1-16.

JONES J. B., B. WOLF, H. A. MILLS. 1991. Plant analysis handbook. A practical sampling, preparation, analysis, and interpretation guide. Micro-Macro Publishing, Inc, Athens.

JONES, M. D. \& S. E. SMITH. 2004. Exploring functional definitions of mycorrhizas: are mycorrhizas always mutualisms? Can. J. Botany. 82: 1089-1109.

JOHNSON, N. C. 2010. Resource stoichiometry elucidates the structure and function of arbuscular mycorrhizas across scales. New Phytol. 185: 631647.

KOIDE, R. T. \& M. LI. 1989. Appropriate controls for vesicular-arbuscular mycorrhiza research. New Phytol. 111: 35-44.

LUGO, M. A. \& M. N. CABELLO. 2002. Native arbuscular mycorrhizal fungi (AMF) from mountain grassland (Cordoba, Argentina) I. Seasonal variation of fungal spore diversity. Mycologia 94: 579-586.

MC GONIGLE, T. P., M. MILLER, D. G. EVANS, G. L. FAIRCHILD, J. A. SWAN. 1990. A method which gives an objective measure of colonization of roots by vesicular-arbuscular mycorrhizal fungi. New Phytol. 115: 495-501.

MILLAR, N. S. \& A. E. BENNETT. 2016. Stressed out symbiotes: hypotheses for the influence of abiotic stress on arbuscular mycorrhizal fungi. Oecologia 182: 625-641.

OMAR M. B., L. BOLLAND, W. A. HEATHER. 1979. A permanent mounting medium for fungi. Bull. Brit. Mycol. Soc. 13: 31-32.

OUAHMANE, L., M. HAFIDI, J. THIOULOUSE, M. DUCOUSSO, M. KISA, A. PRIN, A. GALIANA, A. BOUMEZZOUGH \& R. DUPONNOIS. 2007. Improvement of Cupressus atlantica Gaussen growth by inoculation with native arbuscular mycorrhizal fungi. J. Appl. Microbiol. 103: 683690.

PÉREZ, M., \& C. URCELAY. 2009. Differential growth response to arbuscular mycorrhizal fungi and plant density in two wild plants belonging to contrasting functional types. Mycorrhiza 19: 517-523.

PHILLIPS J. M. \& D. S. HAYMAN. 1970. Improved procedures for clearing roots and staining parasitic and vesicular arbuscular mycorrhizal fungi for rapid assessment of infection. T. Brit. Mycol. Soc. 55: 158-161.

SMITH, S. E., I. JAKOBSEN, M. GRONLUND \& F. A. SMITH. 2011. Roles of arbuscular mycorrhizas in plant phosphorus nutrition: interactions between pathways of phosphorus uptake in arbuscular mycorrhizal roots have important implications for understanding and manipulating plant phosphorus acquisition. Plant Physiology 156: 1050-1057.

SMITH S. E. \& D. READ. 2008. Mycorrhizal symbiosis, $3^{\text {rd }}$ edn. Academic Press, Great Britain.

SCHENK, N. C. \& Y. PEREZ. 1990. Manual of identification of vesicular-arbuscular mycorrhizal fungi. INVAM, University of Florida, Gainesville.

SOTERAS F., D. RENISON \& A. G. BECERRA. 2013. Growth response, phosphorus content and root colonization of Polylepis australis Bitt. seedlings inoculated with different soil types. New Forests 44: 577-589.

SOTERAS, F., D. RENISON \& A. G. BECERRA. 2014. Restoration of high altitude forests in an area affected by a wildfire: Polylepis australis Bitt. seedlings performance after soil inoculation. Trees 28: $173-182$.

SUDOVÁ, R. \& M. VOSÁTKA. 2008. Effects of inoculation with native arbuscular mycorrhizal fungi on clonal growth of Potentilla reptans and Fragaria moschata (Rosaceae). Plant Soil 308: 55-67.

TURNAU, K. \& K. HASELWANDTER. 2002. Arbuscular mycorrhizal fungi, an essential component of soil microflora in ecosystem restoration. In: GIANINAZZI S., H. SCHÜEPP, J. M. BAREA \& K. HASELWANDTER (eds.), Mycorrhizal technology in agriculture, pp. 137-149. Birkhäuser, Verlag/ Switzerland.

VERESOGLOU, S. D., G. MENEXES \& M. C. RILLIG. 2012. Do arbuscular mycorrhizal fungi affect the allometric partition of host plant biomass to shoots and roots? A meta-analysis of studies from 1990 to 2010. Mycorrhiza 22: 227-235.

WALKER, C., W. MIZE, H. S. MCNABB. 1982. Populations of endogonaceous fungi at two populations in central Iowa. Can. J. Botany 60: 2518-2529.

WALKLEY, A. \& I. A. BLACK. 1934. An examination of the Degtjareff method for determining soil organic matter, and a proposed modification of the chromic acid titration method. Soil Sci. 37: 29-38.

WEHNER, J., P. M. ANTUNES, J. R. POWELL, J. MAZUKATOW \& M. C. RILLING. 2010. Plant pathogen protection by arbuscular mycorrhizas: a role for fungal diversity? Pedobiologia 53: 197-201.

Recibido el 7 de septiembre de 2017, aceptado el 19 de febrero de 2018. Editor: Leopoldo Iannone. 
